\title{
Permanence and extinction of regime-switching predator-prey models
}

\author{
Jianhai $\mathrm{Bao}^{b}$, Jinghai Shao ${ }^{a}$ \\ a: School of Mathematical Sciences, Beijing Normal University, Beijing 100875, China \\ b: Department of Mathematics, Central South University, Changsha, Hunan 410083, China
}

January 14, 2020

\begin{abstract}
In this work we study the permanence and extinction of a regime-switching predatorprey model with Beddington-DeAngelis functional response. The switching process is used to describe the random changes of corresponding parameters such as birth and death rates of a species in different environments. When a prey will die out in some fixed environments and will not in the others, our criteria can justify whether it dies out or not in a random switching environment. Our criteria are rather sharp, and they cover the known on-off type results on permanence of predator-prey models without switching. Our method relies on the recent study of ergodicity of regime-switching diffusion processes.
\end{abstract}

AMS subject Classification (2010): 60H10, 92D25, 60J60

Keywords: predator-prey model; extinction; permanence; regime-switching diffusion

\section{Introduction}

In an ecosystem, all species evolve and compete to seek resources to sustain their existence. Denote the two population sizes at time $t$ by $X_{t}$ and $Y_{t}$, respectively. $X_{t}$ denotes the population size of the prey and $Y_{t}$ stands for the population size of the predator. The Kolmogorov predatorprey model is a general deterministic model taking the form:

$$
\left\{\begin{array}{l}
\dot{X}_{t}=X_{t} f\left(X_{t}, Y_{t}\right) \\
\dot{Y}_{t}=Y_{t} g\left(X_{t}, Y_{t}\right)
\end{array}\right.
$$


For $f(x, y)=b-p y$ and $g(x, y)=c x-d$, one gets the well-known Lotka-Volterra model. These deterministic models have been studied extensively. We refer to the monograph [8] due to Hofbauer and Sigmund for a related study on this deterministic model. Here $f(X, Y)$ and $g(X, Y)$ stand for the capita growth rate of each species, which is dependent on the population sizes of both species. Various functional response functions have been used considerably in modeling population dynamics such as Holling types, Hassel-Varley type, Leslie-Gower Holling II type, Beddington-DeAngelis type, etc. However, due to the continuous fluctuation in the environment, the birth rates, death rates, carrying capacities, competition coefficients and all the other parameters involved in this model exhibit random fluctuation to a great extent. To describe this phenomenon, stochastic predator-prey models with different kinds of responses are proposed and there are many works to study these models (cf. [1, [10, [11] and references therein). For a predator-prey system with Beddington-DeAngelis functional response, in practice, we usually estimate the birth rate of the prey and death rate of the predator by their average values plus errors which follow normal distributions. As a result, one gets the following stochastic predatorprey model with Beddington-DeAngelis functional response:

$$
\left\{\begin{array}{l}
\mathrm{d} X_{t}=X_{t}\left(a_{1}-b_{1} X_{t}-\frac{c_{1} Y_{t}}{m_{1}+m_{2} X_{t}+m_{3} Y_{t}}\right) \mathrm{d} t+\alpha X_{t} \mathrm{~d} B_{1}(t), \\
\mathrm{d} Y_{t}=Y_{t}\left(-a_{2}-b_{2} Y_{t}+\frac{c_{2} X_{t}}{m_{1}+m_{2} X_{t}+m_{3} Y_{t}}\right) \mathrm{d} t+\beta Y_{t} \mathrm{~d} B_{2}(t),
\end{array}\right.
$$

with $X_{0}=x_{0}>0$ and $Y_{0}=y_{0}>0$. All the parameters in (1.1) are positive, and $\left(B_{1}(t)\right),\left(B_{2}(t)\right)$ are Brownian motions on the line. For the model (1.1), by Khasminskii's criterion on existence of stationary distributions for diffusion processes (see, e.g., [7, Theorem 4.1]), Ji-Jiang [10] revealed that $\left(X_{t}, Y_{t}\right)$ enjoys a stationary distribution $\mu$, which is also ergodic; Du et al. [6] showed that if $-a_{2}-\frac{\beta^{2}}{2}-\int_{-\infty}^{\infty} \frac{c_{2} \mathrm{e}^{x}}{m_{1}+m_{2} \mathrm{e}^{x}} f^{*}(x) \mathrm{d} x=: \lambda<0$, where $f^{*}(x)=C \exp \left(\frac{2 a_{1}-\alpha^{2}}{\alpha^{2}} x-\frac{2 b_{1}}{\alpha^{2}} \mathrm{e}^{x}\right)$ with $C$ being the normalizing constant, the predator $Y_{t}$ will eventually extinct (i.e. $\lim _{t \rightarrow \infty} Y_{t}=0$ a.s.), and that, in the case $\lambda>0,\left(X_{t}, Y_{t}\right)$ has a unique invariant probability measure $\mu^{*}$ which is supported in $\mathbb{R}_{+}^{2, o}$; Liu-Wang [12] showed that the equilibrium $\left(x^{*}, y^{*}\right)$ is stochastically asymptotically stable in the large (i.e. for any initial data $\left(x_{0}, y_{0}\right), \lim _{t \rightarrow \infty} X_{t}=x^{*}$ a.s. and $\lim _{t \rightarrow \infty} Y_{t}=y^{*}$ a.s.); Li-Zhang [14] provided some sufficient conditions for non-persistence in the mean (i.e. $\lim \sup _{t \rightarrow \infty}\left(\frac{1}{t} \int_{0}^{t} X_{s} \mathrm{~d} s\right)=0$ a.s.), strong persistence in the mean (i.e. $\liminf _{t \rightarrow \infty}\left(\frac{1}{t} \int_{0}^{t} X_{s} \mathrm{~d} s\right)>0$ a.s.), and weak persistence in the mean (i.e. $\limsup _{t \rightarrow \infty}\left(\frac{1}{t} \int_{0}^{t} X_{s} \mathrm{~d} s\right)>0$ a.s.) of the prey population $X_{t}$.

From the viewpoint of biological modeling, variability of the environment may have an important impact on the dynamics of the community. For instance, the distinctive season change such as dry and rainy seasons is observed in monsoon forests, and it characterizes the vegetation there. Also, in boreal and arctic regions, seasonality exerts a strong influence on the dynamics 
of mammals. Moreover, the growth rates, the death rates and the carrying capacities often vary according to the changes in nutrition and food resources. All of these changes usually cannot be described by the traditional deterministic or stochastic predator-prey models. Therefore, it is natural to consider the predator-prey model in a random environment, which is formulated by an additional factor process. More precisely, consider the following regime-switching predator-prey model with Beddington-DeAngelis functional response:

$$
\left\{\begin{array}{l}
\mathrm{d} X_{t}=X_{t}\left(a_{1}\left(\Lambda_{t}\right)-b_{1}\left(\Lambda_{t}\right) X_{t}-\frac{c_{1}\left(\Lambda_{t}\right) Y_{t}}{m_{1}\left(\Lambda_{t}\right)+m_{2}\left(\Lambda_{t}\right) X_{t}+m_{3}\left(\Lambda_{t}\right) Y_{t}}\right) \mathrm{d} t+\alpha\left(\Lambda_{t}\right) X_{t} \mathrm{~d} B_{1}(t) \\
\mathrm{d} Y_{t}=Y_{t}\left(-a_{2}\left(\Lambda_{t}\right)-b_{2}\left(\Lambda_{t}\right) Y_{t}+\frac{c_{2}\left(\Lambda_{t}\right) X_{t}}{m_{1}\left(\Lambda_{t}\right)+m_{2}\left(\Lambda_{t}\right) X_{t}+m_{3}\left(\Lambda_{t}\right) Y_{t}}\right) \mathrm{d} t+\beta\left(\Lambda_{t}\right) Y_{t} \mathrm{~d} B_{2}(t)
\end{array}\right.
$$

with $X_{0}=x_{0}>0$ and $Y_{0}=y_{0}>0$, where $\left(B_{1}(t)\right)$ and $\left(B_{2}(t)\right)$ are Brownian motions on the line, and $\left(\Lambda_{t}\right)$ is a continuous time Markov chain with a finite state space $\mathcal{S}=\{1,2, \ldots, N\}$, $1 \leq N<\infty$. Throughout this paper, the processes $\left(B_{1}(t)\right),\left(B_{2}(t)\right)$ and $\left(\Lambda_{t}\right)$ are defined on a complete probability space $\left(\Omega, \mathscr{F},\left\{\mathscr{F}_{t}\right\}_{t \geq 0}, \mathbb{P}\right)$, and $\left(\Lambda_{t}\right)$ is independent of $\left(B_{1}(t)\right)$ and $\left(B_{2}(t)\right)$. The parameters $a_{k}(\cdot), b_{k}(\cdot), c_{k}(\cdot)$ for $k=1,2$, and $m_{l}(\cdot)$ for $l=1,2,3$, are all positive functions on $\mathcal{S}$.

The dynamical system (1.2) is a regime-switching diffusion process, which has been widely applied in control problems, storage modeling, neuronal activity, biology and mathematical finance. We refer the readers to [2, 4, 19, 20, 22, 23, 24] and the monographs [17, 26] for the study on recurrence, ergodicity, stochastic stability, numerical approximation of regimeswitching diffusion processes with Markovian switching or state-dependent switching in a finite state space or an infinite state space. There is a vast literature on population dynamics with regime switching. For instance, Du-Du [5] described the omega-limit set of Kolmogorov systems of competitive type under the telegraph noise and investigated properties of stationary density; Zhu-Yin [28] examined certain long-run-average limits, and Zhu-Yin [29] investigated long-time behavior of sample paths for competitive Lotka-Volterra ecosystems. In the study of a population system, permanence and extinction are two important and interesting properties, respectively meaning that the population system will survive or die out in the future. Yuan et al. [27] discussed extinction for stochastic hybrid delay population dynamics with $n$ interacting species, and Li et al. [15] and Liu-Wang [13] studied permanence and extinction for stochastic logistic populations with single species.

The regime-switching predator-prey model can describe a very important and interesting situation. We consider a simple example to introduce it. Let us consider the case $\mathcal{S}=\{1,2\}$, where " 1 " denotes the rainy season and " 2 " denotes the dry season. The situation that $a_{1}(1)-$ $\frac{1}{2} \alpha^{2}(1)>0$ and $a_{1}(2)-\frac{1}{2} \alpha^{2}(2)<0$ is rather possible to occur. This means that the birth rate with perturbation of $\left(X_{t}\right)$ in the rainy season makes sure that $\left(X_{t}\right)$ will not die out, but 
that of $\left(X_{t}\right)$ in the dry season makes $\left(X_{t}\right)$ die out. Then a natural question arises: will $\left(X_{t}\right)$ in model (1.2) die out or not? This question is very interesting and represents an essential advantage of model (1.2) over model (1.1). However, the solution of this question is not easy to derive, and so far there are few results of this type on the regime-switching predator-prey model or regime-switching population dynamics. On this topic, we refer the reader to [19] for the explicit examples to see the complexity of the regime-switching diffusion processes, and to [4, 20, 21, 24, 26] for some solutions of this type on ergodicity and stability of regime-switching diffusion processes. In this work, we shall provide a sharp criterion to justify whether $\left(X_{t}\right)$ will die out or not (see Theorem 1.1 below).

Let $\left(q_{i j}\right)_{i, j \in \mathcal{S}}$ be the $Q$-matrix of the Markov chain $\left(\Lambda_{t}\right)$ which means that

$$
\mathbb{P}\left(\Lambda_{t+\delta}=l \mid \Lambda_{t}=k\right)= \begin{cases}q_{k l} \delta+o(\delta), & \text { if } k \neq l, \\ 1+q_{k k} \delta+o(\delta), & \text { if } k=l,\end{cases}
$$

for a sufficiently small $\delta>0$. Throughout this work, the matrix $Q=\left(q_{i j}\right)$ is assumed to be irreducible and conservative, i.e. $q_{k k}=-q_{k}:=-\sum_{j \neq k} q_{k j}<0$. As $\mathcal{S}$ is a finite set and $\left(q_{i j}\right)$ is irreducible, the theory of Markov chains tells us that $\left(\Lambda_{t}\right)$ is ergodic and there exists a unique stationary distribution $\left(\mu_{i}\right)$ for it. To state our main result, we need to introduce two auxiliary processes. Let

$$
\mathrm{d} \varphi_{t}=\varphi_{t}\left(a_{1}\left(\Lambda_{t}\right)-b_{1}\left(\Lambda_{t}\right) \varphi_{t}\right) \mathrm{d} t+\alpha\left(\Lambda_{t}\right) \varphi_{t} \mathrm{~d} B_{1}(t)
$$

and

$$
\mathrm{d} \psi_{t}=\psi_{t}\left(-a_{2}\left(\Lambda_{t}\right)+\frac{c_{2}\left(\Lambda_{t}\right)}{m_{2}\left(\Lambda_{t}\right)}-b_{2}\left(\Lambda_{t}\right) \psi_{t}\right) \mathrm{d} t+\beta\left(\Lambda_{t}\right) \psi_{t} \mathrm{~d} B_{2}(t)
$$

with $\varphi_{0}=X_{0}=x_{0}>0$ and $\psi_{0}=Y_{0}=y_{0}>0$, where $\left(\Lambda_{t}\right),\left(B_{1}(t)\right),\left(B_{2}(t)\right)$ are defined as in (1.2). By the comparison theorem for SDEs (cf. [9]), $\varphi_{t} \geq X_{t}$ and $\psi_{t} \geq Y_{t}$ a.s. for all $t>0$. Our main result of this work is the following theorem.

Theorem 1.1 Let $\left(X_{t}, Y_{t}, \Lambda_{t}\right)$ be defined by (1.2) and (1.3) and $\left(\mu_{i}\right)_{i \in \mathcal{S}}$ be the stationary distribution of the process $\left(\Lambda_{t}\right)$.

(i) If $\sum_{i \in \mathcal{S}} \mu_{i}\left(a_{1}(i)-\frac{1}{2} \alpha^{2}(i)\right)<0$, then $\lim _{t \rightarrow \infty} X_{t}=0$ a.s. , $\lim _{t \rightarrow \infty} Y_{t}=0$ a.s.

(ii) If $\sum_{i \in \mathcal{S}} \mu_{i}\left(a_{1}(i)-\frac{1}{2} \alpha^{2}(i)\right)>0$, then $\left(\varphi_{t}, \Lambda_{t}\right)$ is positive recurrent with stationary distribution $\pi^{\varphi}$ on $\mathbb{R}_{+} \times \mathcal{S}$. Assume further that

$$
\lambda:=-\sum_{i \in \mathcal{S}} \mu_{i}\left(a_{2}(i)+\frac{1}{2} \beta^{2}(i)\right)+\sum_{i \in \mathcal{S}} \int_{\mathbb{R}^{+}} \frac{c_{2}(i) x}{m_{1}(i)+m_{2}(i) x} \pi^{\varphi}(\mathrm{d} x, i)<0 .
$$


Then, $\lim _{t \rightarrow \infty} Y_{t}=0$ a.s., $\limsup _{t \rightarrow \infty} X_{t}>0$ a.s., and the distribution of $\left(X_{t}, \Lambda_{t}\right)$ converges weakly to $\pi^{\varphi}$.

(iii) If $\sum_{i \in \mathcal{S}} \mu_{i}\left(a_{2}(i)+\frac{1}{2} \beta^{2}(i)-\frac{c_{2}(i)}{m_{2}(i)}\right)<0$, then $\left(\psi_{t}, \Lambda_{t}\right)$ is positive recurrent with stationary distribution $\pi^{\psi}$. Assume further that $\sum_{i \in \mathcal{S}} \mu_{i}\left(a_{1}(i)-\frac{1}{2} \alpha^{2}(i)\right)>0$ and

$$
\bar{\lambda}:=\lambda+\sum_{i \in \mathcal{S}} \mu_{i}\left(a_{2}(i)+\frac{1}{2} \beta^{2}(i)-\frac{c_{2}(i)}{m_{2}(i)}\right)+\sum_{i \in \mathcal{S}} \int_{\mathbb{R}_{+}} b_{2}(i) y \pi^{\psi}(\mathrm{d} y, i)>0 .
$$

Then, $\limsup _{t \rightarrow \infty} X_{t}>0$ a.s., $\limsup _{t \rightarrow \infty} Y_{t}>0$ a.s., and $\left(X_{t}, Y_{t}, \Lambda_{t}\right)$ has a stationary distribution.

This theorem will be proved in the next section. As we mentioned, there are few results on permanence for the model (1.2) although there have been numerous works on extinction. Whereas, in Theorem 1.1, we provide a criterion which can justify whether a prey dies out or not when it lives in a random switching environment such that it will die out in some fixed environments and won't die out in others. We shall note that, when $\mathcal{S}$ contains only one state, and hence there is no switching in (1.2) in this case, our result will coincide with the results in 6]. Actually, according to a similar calculation of [6, (2.3)], $\sum_{i \in \mathcal{S}} \mu_{i}\left(a_{2}(i)+\frac{1}{2} \beta^{2}(i)-\frac{c_{2}(i)}{m_{2}(i)}\right)+$ $\sum_{i \in \mathcal{S}} \int_{\mathbb{R}_{+}} b_{2}(i) y \pi^{\psi}(\mathrm{d} y, i)=0$ in the case $N=1$, hence $\bar{\lambda}=\lambda$ in this case. But when $N>1$, we have no way to calculate the precise value of this term at present stage due to lack of explicit representation of the invariant measures of regime-switching diffusion processes. Moreover, we should point out that [14] investigated strong (weak) permanence in the mean for the model (1.1). Compared with [6], the main difficulty in the present work is to determine the recurrence property of stochastic processes $\left(\varphi_{t}, \Lambda_{t}\right)$ and $\left(\psi_{t}, \Lambda_{t}\right)$, which is overcome by using the recent results in [21] on justifying the recurrence property of regime-switching diffusion processes.

The distributions $\pi^{\varphi}$ and $\pi^{\psi}$ are stationary distributions of one-dimensional regime-switching diffusion processes. In [22], for one-dimensional regime-switching diffusion process, an explicit representation of the stationary distribution is provided based on the hitting times of this process.

At last, according to the argument of our main result, the permanence or non-permanence of $\left(X_{t}, Y_{t}\right)$ does not depend on the correlation of the Brownian motions $\left(B_{1}(t)\right)$ and $\left(B_{2}(t)\right)$. So in the present work, we do not impose any condition on the dependence between $\left(B_{1}(t)\right)$ and $\left(B_{2}(t)\right)$. 


\section{Proof of the main result}

We first investigate the properties of the processes $\left(\varphi_{t}, \Lambda_{t}\right)$ and $\left(\psi_{t}, \Lambda_{t}\right)$ including the estimate of their moments and recurrence property. Set

$$
\hat{a}_{1}:=\min _{i \in \mathcal{S}} a_{1}(i), \check{a}_{1}:=\max _{i \in \mathcal{S}} a_{1}(i)
$$

and similarly define $\hat{a}_{2}, \check{a}_{2}, \hat{b}_{k}, \check{b}_{k}, \hat{c}_{k}, \check{c}_{k}$ for $k=1,2$ and $\hat{m}_{l}, \check{m}_{l}$ for $l=1,2,3$. By the finiteness of $\mathcal{S}$, all the parameters introduced here remain positive.

Lemma 2.1 For any $p>1$,

$$
\begin{aligned}
\mathbb{E} \varphi_{t}^{p} \leq & {\left[\left(\mathbb{E} \varphi_{0}^{p}\right)^{-\frac{1}{p}} \exp \left(-\left(\check{a}_{1}+\frac{p-1}{2} \check{\alpha}^{2}\right) t\right)\right.} \\
& \left.+\frac{\hat{b}_{1}}{\check{a}_{1}+\frac{p-1}{2} \check{\alpha}^{2}}\left(1-\exp \left(-\left(\check{a}_{1}+\frac{p-1}{2} \check{\alpha}^{2}\right) t\right)\right)\right]^{-p},
\end{aligned}
$$

and

$$
\begin{aligned}
\mathbb{E} \psi_{t}^{p} \leq & {\left[\left(\mathbb{E} \psi_{0}^{p}\right)^{-\frac{1}{p}} \exp \left(-\left(-\hat{a}_{2}+\frac{\check{c}_{2}}{\hat{m}_{2}}+\frac{p-1}{2} \check{\beta}^{2}\right) t\right)\right.} \\
& \left.+\frac{\hat{b}_{2}}{-\hat{a}_{2}+\frac{\check{c}_{2}}{\dot{m}_{2}}+\frac{p-1}{2} \check{\beta}^{2}}\left(1-\exp \left(-\left(-\hat{a}_{2}+\frac{\check{c}_{2}}{\hat{m}_{2}}+\frac{p-1}{2} \check{\beta}^{2}\right) t\right)\right)\right]^{-p} .
\end{aligned}
$$

Therefore, for any $p>1$,

$$
\limsup _{t \rightarrow \infty} \mathbb{E} \varphi_{t}^{p} \leq\left(\frac{\check{a}_{1}+\frac{p-1}{2} \check{\alpha}^{2}}{\hat{b}_{1}}\right)^{p},
$$

and for any $p>1$ such that $-\hat{a}_{2}+\frac{\check{c}_{2}}{\hat{m}_{2}}+\frac{p-1}{2} \check{\beta}^{2}>0$, it holds

$$
\limsup _{t \rightarrow \infty} \mathbb{E} \psi_{t}^{p} \leq\left(\frac{-\hat{a}_{2}+\frac{\check{c}_{2}}{\hat{m}_{2}}+\frac{p-1}{2} \check{\beta}^{2}}{\hat{b}_{2}}\right)^{p} .
$$

Proof. We shall only prove the estimate for $\varphi_{t}$ since the estimate for $\psi_{t}$ can be done in the same way. By Itô's formula,

$$
\begin{aligned}
\mathrm{d} \varphi_{t}^{p} & =p\left(a_{1}\left(\Lambda_{t}\right)+\frac{p-1}{2} \alpha^{2}\left(\Lambda_{t}\right)\right) \varphi_{t}^{p} \mathrm{~d} t-p b_{1}\left(\Lambda_{t}\right) \varphi_{t}^{p+1} \mathrm{~d} t+p \alpha\left(\Lambda_{t}\right) \varphi_{t}^{p} \mathrm{~d} B_{1}(t) \\
& \leq p\left(\check{a}_{1}+\frac{p-1}{2} \check{\alpha}^{2}\right) \varphi_{t}^{p} \mathrm{~d} t-p \hat{b}_{1} \varphi_{t}^{p+1} \mathrm{~d} t+p \alpha\left(\Lambda_{t}\right) \varphi_{t}^{p} \mathrm{~d} B_{1}(t) .
\end{aligned}
$$


Taking the expectation on both sides and utilizing Hölder's inequality yields that

$$
\begin{aligned}
\frac{\mathrm{d} \mathbb{E} \varphi_{t}^{p}}{\mathrm{~d} t} & \leq p\left(\check{a}_{1}+\frac{p-1}{2} \check{\alpha}^{2}\right) \mathbb{E} \varphi_{t}^{p}-p \hat{b}_{1} \mathbb{E} \varphi_{t}^{p+1} \\
& \leq p\left(\check{a}_{1}+\frac{p-1}{2} \check{\alpha}^{2}\right) \mathbb{E} \varphi_{t}^{p}-p \hat{b}_{1}\left(\mathbb{E} \varphi_{t}^{p}\right)^{\frac{p+1}{p}} .
\end{aligned}
$$

By the comparison theorem for ordinary differential equations, we get (2.2), and then (2.4) follows immediately.

Note that (2.5) holds for $p$ sufficiently large. By Lemma 2.1, the processes $\left(\varphi_{t}, \Lambda_{t}\right)$ and $\left(\psi_{t}, \Lambda_{t}\right)$ are nonexplosive, and hence the processes $\left(X_{t}, \Lambda_{t}\right)$ and $\left(Y_{t}, \Lambda_{t}\right)$ are also nonexplosive due to $\varphi_{t} \geq X_{t}$ a.s. and $\psi_{t} \geq Y_{t}$ a.s. for all $t>0$.

Lemma 2.2 It holds that

$$
\mathbb{P}\left(X_{t}>0, \forall t>0\right)=1 \quad \text { and } \quad \mathbb{P}\left(Y_{t}>0, \forall t>0\right)=1 .
$$

Proof. Here we only consider the process $\left(X_{t}\right)$ while the result for $\left(Y_{t}\right)$ can be dealt with in a similar manner. Let

$$
\tau_{\Delta}=\inf \left\{t>0 ; X_{t} \leq \Delta\right\}, \Delta>0 ; \quad \sigma_{K}=\inf \left\{t>0 ; X_{t} \geq K\right\}, \quad K \geq 1 .
$$

Let $\tau_{0}=\inf \left\{t>0 ; X_{t}=0\right\}$, then $\tau_{\Delta} \uparrow \tau_{0}$ as $\Delta \downarrow 0$. As $\left(X_{t}, \Lambda_{t}\right)$ is nonexplosive, $\lim _{K \rightarrow \infty} \sigma_{K}=\infty$ a.s. By Itô's formula, for $p>0$ such that $-\hat{a}_{1}+\frac{\check{c}_{1}}{\hat{m}_{3}}+\frac{p+1}{2} \check{\alpha}^{2}>0$, we have

$$
\begin{aligned}
\mathbb{E} X_{t \wedge \sigma_{K} \wedge \tau_{\Delta}}^{-p}=x_{0}^{-p}+p \mathbb{E} \int_{0}^{t \wedge \sigma_{K} \wedge \tau_{\Delta}}\left(-a_{1}\left(\Lambda_{s}\right)+\frac{p+1}{2} \alpha^{2}\left(\Lambda_{s}\right)\right. \\
\left.+\frac{c_{1}\left(\Lambda_{s}\right) Y_{s}}{m_{1}\left(\Lambda_{s}\right)+m_{2}\left(\Lambda_{s}\right) X_{s}+m_{3}\left(\Lambda_{s}\right) Y_{s}}+b_{1}\left(\Lambda_{s}\right) X_{s}\right) X_{s}^{-p} \mathrm{~d} s \\
\leq x_{0}^{-p}+p \mathbb{E} \int_{0}^{t \wedge \sigma_{K} \wedge \tau_{\Delta}}\left(-\hat{a}_{1}+\frac{\check{c}_{1}}{\hat{m}_{3}}+\frac{p+1}{2} \check{\alpha}^{2}+\check{b}_{1} K\right) X_{s}^{-p} \mathrm{~d} s .
\end{aligned}
$$

By Gronwall's inequality,

$$
\mathbb{E} X_{t \wedge \sigma_{K} \wedge \tau_{\Delta}}^{-p} \leq x_{0}^{-p} \exp \left(p\left(-\hat{a}_{1}+\frac{\check{c}_{1}}{\hat{m}_{3}}+\frac{p+1}{2} \check{\alpha}^{2}+\check{b}_{1} K\right) t\right) .
$$

If $\mathbb{P}\left(\tau_{0}<\infty\right)>0$, we can choose $t, K$ large enough so that $\mathbb{P}\left(\tau_{0}<t \wedge \sigma_{K}\right)>0$. Then for any $\Delta>0$,

$$
\begin{aligned}
0 & <\mathbb{P}\left(\tau_{0}<t \wedge \sigma_{K}\right) \leq \mathbb{P}\left(\tau_{\Delta}<t \wedge \sigma_{K}\right) \\
& \leq \Delta^{p} \mathbb{E}\left[X_{t \wedge \sigma_{K} \wedge \tau_{\Delta}}^{-p} \mathbf{1}_{\tau_{\Delta}<t \wedge \sigma_{K}}\right] \leq \Delta^{p} \mathbb{E}\left[X_{t \wedge \sigma_{K} \wedge \tau_{\Delta}}^{-p}\right] \\
& \leq \Delta^{p} x_{0}^{-p} \exp \left(p\left(-\hat{a}_{1}+\frac{\check{c}_{1}}{\hat{m}_{3}}+\frac{p+1}{2} \check{\alpha}^{2}+\check{b}_{1} K\right) t\right) .
\end{aligned}
$$


Taking $\Delta \downarrow 0$, we get a contradiction. Hence $\mathbb{P}\left(\tau_{0}<\infty\right)=0$ and we complete the proof.

Next, we go to study the recurrence property of the processes $\left(\varphi_{t}, \Lambda_{t}\right)$ and $\left(\psi_{t}, \Lambda_{t}\right)$, which plays a fundamental role in the proof of our Theorem 1.1.

Lemma 2.3 (Key lemma) (i) When $\sum_{i \in \mathcal{S}} \mu_{i}\left(a_{1}(i)-\frac{1}{2} \alpha^{2}(i)\right)>0$, the process $\left(\varphi_{t}, \Lambda_{t}\right)$ is positive recurrent and has a unique stationary distribution $\pi^{\varphi}$, which is a probability measure on $(0, \infty) \times \mathcal{S}$. When $\sum_{i \in \mathcal{S}} \mu_{i}\left(a_{1}(i)-\frac{1}{2} \alpha^{2}(i)\right)<0$, the process $\left(\varphi_{t}, \Lambda_{t}\right)$ is transient.

(ii) When $\sum_{i \in \mathcal{S}} \mu_{i}\left(a_{2}(i)+\frac{1}{2} \beta^{2}(i)-\frac{c_{2}(i)}{m_{2}(i)}\right)<0$, the process $\left(\psi_{t}, \Lambda_{t}\right)$ is positive recurrent and has a unique stationary distribution $\pi^{\psi}$, which is a probability measure on $(0, \infty) \times \mathcal{S}$. When $\sum_{i \in \mathcal{S}} \mu_{i}\left(a_{2}(i)+\frac{1}{2} \beta^{2}(i)-\frac{c_{2}(i)}{m_{2}(i)}\right)>0$, the process $\left(\psi_{t}, \Lambda_{t}\right)$ is transient.

Proof. (1) We shall apply the method adopted in the argument of [21, Theorem 3.1] to prove this lemma. As the diffusion coefficient of $\varphi_{t}$ is degenerate at the point $x=0$, we use the transform $Z_{t}=\ln \varphi_{t}$. By Lemma 2.2, this transform makes sense for all $t \geq 0$ a.s. Then $Z_{t}$ satisfies the following SDE:

$$
\mathrm{d} Z_{t}=\left(a_{1}\left(\Lambda_{t}\right)-\frac{1}{2} \alpha^{2}\left(\Lambda_{t}\right)-b_{1}\left(\Lambda_{t}\right) \mathrm{e}^{Z_{t}}\right) \mathrm{d} t+\alpha\left(\Lambda_{t}\right) \mathrm{d} B_{1}(t)
$$

The recurrence property of $\left(\varphi_{t}, \Lambda_{t}\right)$ is equivalent to that of $\left(Z_{t}, \Lambda_{t}\right)$. For each $i \in \mathcal{S}$, set $L^{(i)}:=$ $\left(a_{1}(i)-\frac{1}{2} \alpha^{2}(i)-b_{1}(i) \mathrm{e}^{x}\right) \frac{\mathrm{d}}{\mathrm{d} x}+\frac{1}{2} \alpha^{2}(i) \frac{\mathrm{d}^{2}}{\mathrm{~d} x^{2}}$. Then the operator $\mathscr{A}$ defined by

$$
\mathscr{A} f(x, i)=L^{(i)} f(\cdot, i)(x)+\sum_{j \neq i} q_{i j}(f(x, j)-f(x, i)), \quad f \in C^{2}(\mathbb{R} \times \mathcal{S})
$$

is the infinitesimal generator of the process $\left(Z_{t}, \Lambda_{t}\right)$.

Set $\beta_{i}:=a_{1}(i)-\frac{1}{2} \alpha^{2}(i), i \in \mathcal{S}$. We first show the positive recurrence of $\left(Z_{t}, \Lambda_{t}\right)$. Take $h(x)=x^{2}$ and $g(x)=|x|$. Then

$$
L^{(i)} h(x)=2\left(\beta_{i}-b_{1}(i) \mathrm{e}^{x}+\frac{1}{2} \alpha^{2}(i) x^{-1}\right) x, \quad \text { for }|x|>0 .
$$

Note that $\lim _{x \rightarrow+\infty}-b_{1}(i) \mathrm{e}^{x}=-\infty$, and $\lim _{x \rightarrow-\infty}-b_{1}(i) \mathrm{e}^{x}=0$. Due to $\sum_{i \in \mathcal{S}} \mu_{i} \beta_{i}>0$, there exist $\varepsilon>0$ and $r_{0}>0$ such that $\sum_{i \in \mathcal{S}} \mu_{i}\left(\beta_{i}-\varepsilon\right)>0$ and

$$
\begin{gathered}
2\left(\beta_{i}-b_{1}(i) \mathrm{e}^{x}+\frac{1}{2} \alpha^{2}(i) x^{-1}\right) x \leq-2\left(\beta_{i}-\varepsilon\right) g(x), \quad \text { for } x>r_{0}, i \in \mathcal{S} . \\
2\left(\beta_{i}-b_{1}(i) \mathrm{e}^{x}+\frac{1}{2} \alpha^{2}(i) x^{-1}\right) x \leq-2\left(\beta_{i}-\varepsilon\right) g(x), \quad \text { for } x<-r_{0}, i \in \mathcal{S} .
\end{gathered}
$$


Thanks to $-\sum_{i \in \mathcal{S}} \mu_{i}\left(\beta_{i}-\varepsilon\right)<0$, by the Fredholm alternative (cf. [18, pp.434]), there exist a constant $\kappa>0$ and a vector $\boldsymbol{\xi}$ such that

$$
Q \boldsymbol{\xi}(i)=-\kappa+2\left(\beta_{i}-\varepsilon\right), \quad i \in \mathcal{S}
$$

Set $V(x, i):=h(x)+\xi_{i} g(x)$. We have

$$
\begin{aligned}
\mathscr{A} V(x, i) & =L^{(i)} h(x)+\xi_{i} L^{(i)} g(x)+Q \boldsymbol{\xi}(i) g(x) \\
& \leq\left(-2\left(\beta_{i}-\varepsilon\right)+Q \boldsymbol{\xi}(i)+\frac{L^{(i)} g(x)}{g(x)} \xi_{i}\right) g(x) \\
& =\left(-\kappa+\frac{L^{(i)} g(x)}{g(x)} \xi_{i}\right) g(x), \quad|x|>r_{0} .
\end{aligned}
$$

As $\lim _{x \rightarrow+\infty} \frac{L^{(i)} g(x)}{g(x)}=-\infty, \lim _{x \rightarrow-\infty} \frac{L^{(i)} g(x)}{g(x)}=0$, and $\lim _{|x| \rightarrow+\infty} g(x)=+\infty$, there exists a constant $r_{1}>r_{0}$ such that

$$
\mathscr{A} V(x, i)<-1, \quad|x|>r_{1}, i \in \mathcal{S}
$$

Consequently, by taking $\lim _{|x| \rightarrow \infty} h(x)=\infty$ into consideration, $\left(Z_{t}, \Lambda_{t}\right)$ and hence $\left(\varphi_{t}, \Lambda_{t}\right)$ are positive recurrent. By [26, Theorem 4.3, pp.114], $\left(Z_{t}, \Lambda_{t}\right)$ has a unique stationary distribution on $\mathbb{R} \times \mathcal{S}$. So the stationary distribution $\pi^{\varphi}$ of $\left(\varphi_{t}, \Lambda_{t}\right)$ is a probability measure on $(0, \infty) \times \mathcal{S}$.

Next, we prove the transience of $\left(Z_{t}, \Lambda_{t}\right)$ under the condition that $\sum_{i \in \mathcal{S}} \mu_{i} \beta_{i}<0$. Since the behavior of $Z_{t}$, caused by the term $-b_{1}(i) e^{x}$, is quite different on $(0,+\infty)$ and $(-\infty, 0)$, we prove the transience property by it's definition.

Let $h(x)=\frac{1}{|x|}$ and $g(x)=h^{\prime}(x)$ for $|x|>0$. Then

$$
L^{(i)} h(x)=\left(\beta_{i}-b_{1}(i) \mathrm{e}^{x}-\frac{\alpha^{2}(i)}{x}\right) \frac{1}{x^{2}}, \quad \text { for } x<0, i \in \mathcal{S} .
$$

In view of

$$
\lim _{x \rightarrow-\infty}\left(\beta_{i}-b_{1}(i) \mathrm{e}^{x}-\frac{\alpha^{2}(i)}{x}\right)=\beta_{i},
$$

there exist $\varepsilon>0, r_{1}>0$ so that

$$
\sum_{i \in \mathcal{S}} \mu_{i}\left(\beta_{i}+\varepsilon\right)<0, \quad \text { and } \beta_{i}-b_{1}(i) \mathrm{e}^{x}-\frac{\alpha^{2}(i)}{x}<\beta_{i}+\varepsilon, \forall x<-r_{1}, i \in \mathcal{S} .
$$

Owing to $\sum_{i \in \mathcal{S}} \mu_{i}\left(\beta_{i}+\varepsilon\right)<0$, the Fredholm alternative yields that there exist a constant $\kappa>0$ and a vector $\boldsymbol{\xi}$ such that

$$
Q \boldsymbol{\xi}(i)=-\kappa-\beta_{i}-\varepsilon
$$


Set $V(x, i):=h(x)+\xi_{i} g(x)$ for $x<0$. We derive that

$$
\begin{aligned}
\mathscr{A} V(x, i) & =L^{(i)} h(x)+\xi_{i} L^{(i)} g(x)+Q \boldsymbol{\xi}(i) g(x) \\
& \leq\left(\beta_{i}+\varepsilon+\xi_{i} \frac{L^{(i)} g(x)}{g(x)}+Q \boldsymbol{\xi}(i)\right) g(x) \\
& =\left(-\kappa+\xi_{i} \frac{L^{(i)} g(x)}{g(x)}\right) g(x), \quad \text { for } x<-r_{1}, i \in \mathcal{S} .
\end{aligned}
$$

It is easy to see that $\lim _{x \rightarrow-\infty} \frac{L^{(i)} g(x)}{g(x)}=0$. Denote by $\xi_{\min }:=\min \left\{\xi_{i} ; i \in \mathcal{S}\right\}$ and $\xi_{\max }:=$ $\max \left\{\xi_{i} ; i \in \mathcal{S}\right\}$. Hence, there exists a constant $r_{2}$ with $r_{2}>r_{1}>0$ such that $h(x)+\xi_{\min } g(x)$ is an increasing function on $\left(-\infty,-r_{2}\right]$ and

$$
\mathscr{A} V(x, i) \leq 0, \quad \text { for } x \leq-r_{2}, i \in \mathcal{S}
$$

Now, take $Z_{0}=z<-r_{2}<0$ such that

$$
h(z)+\xi_{\max } g(z)<h\left(-r_{2}\right)+\xi_{\min } g\left(-r_{2}\right) .
$$

Set

$$
\tau_{-K}:=\inf \left\{t>0 ; Z_{t}=-K\right\}, \quad \tau:=\inf \left\{t>0 ; Z_{t}=-r_{2}\right\} .
$$

By Dynkin's formula, we have

$$
\begin{aligned}
\mathbb{E}\left[V\left(Z_{t \wedge \tau_{-K} \wedge \tau}, \Lambda_{t \wedge \tau_{-K} \wedge \tau}\right)\right] & =V\left(z, i_{0}\right)+\mathbb{E} \int_{0}^{t \wedge \tau_{-K} \wedge \tau} \mathscr{A} V\left(Z_{s}, \Lambda_{s}\right) \mathrm{d} s \\
& \leq V\left(z, i_{0}\right),
\end{aligned}
$$

where $\Lambda_{0}=i_{0}$. Letting $t \rightarrow \infty$, we get

$$
\mathbb{E}\left[V\left(-K, \Lambda_{\tau_{-K}}\right) \mathbf{1}_{\tau_{-K} \leq \tau}\right]+\mathbb{E}\left[V\left(-r_{2}, \Lambda_{\tau}\right) \mathbf{1}_{\tau<\tau_{-K}}\right] \leq V\left(z, i_{0}\right),
$$

which further yields that

$$
\begin{gathered}
\left(h(-K)+\xi_{\min } g(-K)\right) \mathbb{P}\left(\tau \geq \tau_{-K}\right)+\left(h\left(-r_{2}\right)+\xi_{\min } g\left(-r_{2}\right)\right) \mathbb{P}\left(\tau<\tau_{-K}\right) \leq V\left(z, i_{0}\right), \\
\mathbb{P}\left(\tau \geq \tau_{-K}\right) \geq \frac{h(z)+\xi_{\max } g(z)-h\left(-r_{2}\right)-\xi_{\min } g\left(-r_{2}\right)}{h(-K)+\xi_{\min } g(-K)-h\left(-r_{2}\right)-\xi_{\min } g\left(-r_{2}\right)}>0,
\end{gathered}
$$

where in the last step we have used the increasing property of $x \mapsto h(x)+\xi_{\min } g(x)$ on $\left(-\infty,-r_{2}\right]$ and (2.11). Due to Lemma 2.2, and $\varphi_{t} \geq X_{t}$ a.s., we obtain $\tau_{-K} \rightarrow \infty$ a.s. as $K \rightarrow \infty$. Consequently, by passing to the limit as $K \rightarrow \infty$, the previous inequality yields

$$
\mathbb{P}(\tau=\infty)>0,
$$


which implies that the process $\left(Z_{t}, \Lambda_{t}\right)$ is transient, hence $\left(\varphi_{t}, \Lambda_{t}\right)$ is transient too.

(2) The results of $\left(\psi_{t}, \Lambda_{t}\right)$ can be proved by the method analogous to that used above for the process $\left(\psi_{t}, \Lambda_{t}\right)$, which is omitted.

Proposition 2.4 (Strong ergodicity theorem) Assume that $\left(\varphi_{t}, \Lambda_{t}\right)$ and $\left(\psi_{t}, \Lambda_{t}\right)$ are positive recurrent. Let $f$ be a bounded measurable function on $\mathbb{R} \times \mathcal{S}$. Then almost surely

$$
\lim _{t \rightarrow \infty} \frac{1}{t} \int_{0}^{t} f\left(\varphi_{s}, \Lambda_{s}\right) \mathrm{d} s=\sum_{i \in \mathcal{S}} \int_{\mathbb{R}_{+}} f(x, i) \pi^{\varphi}(\mathrm{d} x, i),
$$

and

$$
\lim _{t \rightarrow \infty} \frac{1}{t} \int_{0}^{t} f\left(\psi_{s}, \Lambda_{s}\right) \mathrm{d} s=\sum_{i \in \mathcal{S}} \int_{\mathbb{R}_{+}} f(y, i) \pi^{\psi}(\mathrm{d} y, i) .
$$

Moreover,

$$
\liminf _{t \rightarrow \infty} \frac{1}{t} \int_{0}^{t} b_{1}\left(\Lambda_{s}\right) \varphi_{s} \mathrm{~d} s \geq \sum_{i \in \mathcal{S}} \int_{\mathbb{R}_{+}} b_{1}(i) x \pi^{\varphi}(\mathrm{d} x, i), \quad \text { a.s. }
$$

and

$$
\liminf _{t \rightarrow \infty} \frac{1}{t} \int_{0}^{t} b_{2}\left(\Lambda_{s}\right) \psi_{s} \mathrm{~d} s \geq \sum_{i \in \mathcal{S}} \int_{\mathbb{R}_{+}} b_{2}(i) y \pi^{\psi}(\mathrm{d} y, i), \quad \text { a.s. }
$$

Proof. Similar to [26, Theorem 4.4], one can use the idea of [25, Theorem 3.16, pp.46] to prove (2.12) and (2.13) for regime-switching diffusion processes.

Applying (2.12) to the function $f(z, k)=b_{1}(k) \min \{z, M\}$ for a positive constant $M$, we get

$$
\lim _{t \rightarrow \infty} \frac{1}{t} \int_{0}^{t} b_{1}\left(\Lambda_{s}\right) \min \left\{\varphi_{s}, M\right\} \mathrm{d} s=\sum_{k \in \mathcal{S}} \int_{\mathbb{R}_{+}} b_{1}(k) \min \{z, M\} \pi^{\varphi}(\mathrm{d} z, k) \quad \text { a.s. }
$$

Then

$$
\begin{aligned}
\liminf _{t \rightarrow \infty} \frac{1}{t} \int_{0}^{t} b_{1}\left(\Lambda_{s}\right) \varphi_{s} \mathrm{~d} s & \geq \lim _{t \rightarrow \infty} \frac{1}{t} \int_{0}^{t} b_{1}\left(\Lambda_{s}\right) \min \left\{\varphi_{s}, M\right\} \mathrm{d} s \\
& =\sum_{k \in \mathcal{S}} \int_{\mathbb{R}_{+}} b_{1}(k) \min \{z, M\} \pi^{\varphi}(\mathrm{d} z, k) \quad \text { a.s. }
\end{aligned}
$$

Letting $M$ tend to $\infty$ in the previous inequality, we obtain (2.14). The proof is completed.

After the preparations of the above results on the auxiliary processes $\left(\varphi_{t}\right)$ and $\left(\psi_{t}\right)$, we are ready to prove our main result. As the proof of Theorem 1.1 is a little bit long, we divide it into three propositions. 
Proposition 2.5 If $\sum_{i \in \mathcal{S}} \mu_{i}\left(a_{1}(i)-\frac{1}{2} \alpha^{2}(i)\right)<0$, then almost surely $\lim _{t \rightarrow \infty} X_{t}=0$ and $\lim _{t \rightarrow \infty} Y_{t}=0$.

Proof. By Itô's formula, we have

$$
\begin{aligned}
\mathrm{d} \ln X_{t} & =\left(a_{1}\left(\Lambda_{t}\right)-\frac{1}{2} \alpha^{2}\left(\Lambda_{t}\right)-b_{1}\left(\Lambda_{t}\right) X_{t}-\frac{c_{1}\left(\Lambda_{t}\right) Y_{t}}{m_{1}\left(\Lambda_{t}\right)+m_{2}\left(\Lambda_{t}\right) X_{t}+m_{3}\left(\Lambda_{t}\right) Y_{t}}\right) \mathrm{d} t+\alpha\left(\Lambda_{t}\right) \mathrm{d} B_{1}(t) \\
& \leq\left(a_{1}\left(\Lambda_{t}\right)-\frac{1}{2} \alpha^{2}\left(\Lambda_{t}\right)\right) \mathrm{d} t+\alpha\left(\Lambda_{t}\right) \mathrm{d} B_{1}(t)=: \mathrm{d} \ln \tilde{\varphi}_{t} .
\end{aligned}
$$

By the comparison theorem for stochastic differential equations (cf. [9]), we have

$$
\ln X_{t} \leq \ln \tilde{\varphi}_{t} \text { for any } t>0 \text { a.s. }
$$

Consequently,

$$
\frac{\ln X_{t}}{t} \leq \frac{\ln \tilde{\varphi}_{t}}{t}=\frac{\ln x_{0}}{t}+\frac{1}{t} \int_{0}^{t}\left(a_{1}\left(\Lambda_{s}\right)-\frac{1}{2} \alpha^{2}\left(\Lambda_{s}\right)\right) \mathrm{d} s+\frac{1}{t} \int_{0}^{t} \alpha\left(\Lambda_{s}\right) \mathrm{d} B_{1}(s) .
$$

By the strong ergodicity theorem,

$$
\lim _{t \rightarrow \infty}\left\{\frac{1}{t} \int_{0}^{t}\left(a_{1}\left(\Lambda_{s}\right)-\frac{1}{2} \alpha^{2}\left(\Lambda_{s}\right)\right) \mathrm{d} s+\frac{1}{t} \int_{0}^{t} \alpha\left(\Lambda_{s}\right) \mathrm{d} B_{1}(s)\right\}=\sum_{i \in \mathcal{S}} \mu_{i}\left(a_{1}(i)-\frac{1}{2} \alpha^{2}(i)\right) \quad \text { a.s. }
$$

Here we have used the fact that

$$
\lim _{t \rightarrow \infty} \frac{1}{t} \int_{0}^{t} \alpha\left(\Lambda_{s}\right) \mathrm{d} B_{1}(s)=0 \quad \text { a.s. }
$$

which is due to the boundedness of $(\alpha(k))$ and the strong law of large numbers (cf. [16, Theorem 1.3.4]). Therefore, when $\sum_{i \in \mathcal{S}} \mu_{i}\left(a_{1}(i)-\frac{1}{2} \alpha^{2}(i)\right)<0$, we have $\lim \sup _{t \rightarrow \infty} \frac{\ln X_{t}}{t}<0$ a.s., which further implies that $\lim _{t \rightarrow \infty} X_{t}=0$ a.s.

With $\lim _{t \rightarrow \infty} X_{t}=0$ a.s. in hand, we shall show that $\lim _{t \rightarrow \infty} Y_{t}=0$ a.s. Namely, when the prey $\left(X_{t}\right)$ dies out, the predator $\left(Y_{t}\right)$ also dies out. By Itô's formula, it holds

$$
\begin{aligned}
\limsup _{t \rightarrow \infty} \frac{\ln Y_{t}}{t} & \leq \limsup _{t \rightarrow \infty}\left[\frac{\ln y_{0}}{t}+\frac{1}{t} \int_{0}^{t}\left(-a_{2}\left(\Lambda_{s}\right)-\frac{\beta^{2}\left(\Lambda_{s}\right)}{2}-\frac{c_{2}\left(\Lambda_{s}\right) X_{s}}{m_{1}\left(\Lambda_{s}\right)}\right) \mathrm{d} s+\frac{1}{t} \int_{0}^{t} \beta\left(\Lambda_{s}\right) \mathrm{d} B_{2}(s)\right] \\
& =-\sum_{i \in \mathcal{S}} \mu_{i}\left(a_{2}(i)+\frac{1}{2} \beta^{2}(i)\right)<0, \quad \text { a.s. }
\end{aligned}
$$

which yields immediately that $\lim _{t \rightarrow \infty} Y_{t}=0$ a.s. The proof is therefore completed.

Proposition 2.6 If $\sum_{i \in \mathcal{S}} \mu_{i}\left(a_{1}(i)-\frac{1}{2} \alpha^{2}(i)\right)>0$, and $\lambda<0$, where $\lambda$ is defined by (1.6), then almost surely $\lim _{t \rightarrow \infty} Y_{t}=0$, $\limsup _{t \rightarrow \infty} X_{t}>0$, and the distribution of $\left(X_{t}, \Lambda_{t}\right)$ converges weakly to $\pi^{\varphi}$. 
Proof. We first show that $\left(Y_{t}\right)$ tends to be extinct. By Itô's formula, we have

$\mathrm{d} \ln Y_{t}=\left(-a_{2}\left(\Lambda_{t}\right)-\frac{1}{2} \beta^{2}\left(\Lambda_{t}\right)-b_{2}\left(\Lambda_{t}\right) Y_{t}+\frac{c_{2}\left(\Lambda_{t}\right) X_{t}}{m_{1}\left(\Lambda_{t}\right)+m_{2}\left(\Lambda_{t}\right) X_{t}+m_{3}\left(\Lambda_{t}\right) Y_{t}}\right) \mathrm{d} t+\beta\left(\Lambda_{t}\right) \mathrm{d} B_{2}(t)$.

Since $X_{t} \leq \varphi_{t}$ a.s. for any $t \geq 0$ and $\left(\varphi_{t}, \Lambda_{t}\right)$ is positive recurrent thanks to Lemma 2.3, in addition to the comparison theorem and Proposition 2.4, we obtain that

$$
\begin{aligned}
\limsup _{t \rightarrow \infty} \frac{1}{t} \ln Y_{t} \leq & \limsup _{t \rightarrow \infty} \frac{1}{t} \int_{0}^{t}\left(-a_{2}\left(\Lambda_{s}\right)-\frac{1}{2} \beta^{2}\left(\Lambda_{s}\right)+\frac{c_{2}\left(\Lambda_{s}\right) \varphi_{s}}{m_{1}\left(\Lambda_{s}\right)+m_{2}\left(\Lambda_{s}\right) \varphi_{s}}\right) \mathrm{d} s \\
& +\limsup _{t \rightarrow \infty} \frac{1}{t} \int_{0}^{t} \beta\left(\Lambda_{s}\right) \mathrm{d} B_{2}(s) \\
= & \lambda<0, \quad \text { a.s. }
\end{aligned}
$$

Therefore, $\lim _{t \rightarrow \infty} Y_{t}=0$ a.s.

Next, as $\lim _{t \rightarrow \infty} Y_{t}=0$ a.s., for any $\varepsilon>0$ there exist a measurable subset $\Omega_{\varepsilon} \subset \Omega$ with $\mathbb{P}\left(\Omega_{\varepsilon}\right)>1-\varepsilon$ and a positive constant $t(\varepsilon)$ such that for any $t>t(\varepsilon), \omega \in \Omega_{\varepsilon}$,

$$
\mathrm{d} X_{t} \geq X_{t}\left(a_{1}\left(\Lambda_{t}\right)-b_{1}\left(\Lambda_{t}\right) X_{t}-\frac{c_{1}\left(\Lambda_{t}\right) \varepsilon}{m_{1}\left(\Lambda_{t}\right)+m_{2}\left(\Lambda_{t}\right) X_{t}+m_{3}\left(\Lambda_{t}\right) \varepsilon}\right) \mathrm{d} t+\alpha\left(\Lambda_{t}\right) X_{t} \mathrm{~d} B_{1}(t),
$$

which yields that the distribution of $\left(X_{t}, \Lambda_{t}\right)$ converges weakly to the stationary distribution $\pi^{\varphi}$ of $\left(\varphi_{t}, \Lambda_{t}\right)$ as $t \rightarrow \infty$. Indeed, let $\left(\varphi_{t}^{\varepsilon}\right)$ satisfy

$$
\mathrm{d} \varphi_{t}^{\varepsilon}=\varphi_{t}^{\varepsilon}\left(a_{1}\left(\Lambda_{t}\right)-b_{1}\left(\Lambda_{t}\right) \varphi_{t}^{\varepsilon}-\frac{c_{1}\left(\Lambda_{t}\right) \varepsilon}{m_{1}\left(\Lambda_{t}\right)+m_{2}\left(\Lambda_{t}\right) \varphi_{t}^{\varepsilon}+m_{3}\left(\Lambda_{t}\right) \varepsilon}\right) \mathrm{d} t+\alpha\left(\Lambda_{t}\right) \varphi_{t}^{\varepsilon} \mathrm{d} B_{1}(t),
$$

with $\varphi_{0}^{\varepsilon}=x_{0}$. For any bounded increasing continuous functions $f$ on $\mathbb{R}$, we have

$$
\mathbb{E} f\left(\varphi_{t}\right) \geq \mathbb{E} f\left(X_{t}\right) \geq \mathbb{E}\left[f\left(X_{t}\right) \mathbf{1}_{\Omega_{\varepsilon}}\right]-\varepsilon\|f\| \geq \mathbb{E}\left[f\left(\varphi_{t}^{\varepsilon}\right) \mathbf{1}_{\Omega_{\varepsilon}}\right]-\varepsilon\|f\|
$$

where $\|f\|=\sup _{x}|f(x)|<\infty$. Letting $\varepsilon \rightarrow 0$ and then $t \rightarrow \infty$, we get

$$
\lim _{t \rightarrow \infty} \mathbb{E} f\left(X_{t}\right)=\sum_{i \in \mathcal{S}} \int_{\mathbb{R}_{+}} f(x) \pi^{\varphi}(\mathrm{d} x, i) .
$$

The linearity in the previous equation implies that it also holds for every bounded decreasing continuous functions, hence for every bounded variation functions. Then it is easy to see that the distribution of $\left(X_{t}, \Lambda_{t}\right)$ converges weakly to $\pi^{\varphi}$ as desired.

To complete the proof, we also need to show that $\limsup _{t \rightarrow \infty} X_{t}>0$ a.s. Indeed, if $\mathbb{P}\left(\lim _{t \rightarrow \infty} X_{t}=0\right)>0$, there exists a measurable subset $\Omega_{0}$ of $\Omega$ such that $\mathbb{P}\left(\Omega_{0}\right)>0$ and 
$\forall \omega \in \Omega_{0}, \lim _{t \rightarrow \infty} X_{t}(\omega)=0$. For any $\delta>0$, as the distribution of $\left(X_{t}, \Lambda_{t}\right)$ converges weakly to $\pi^{\varphi}$, we have

$$
\pi^{\varphi}([0, \delta] \times \mathcal{S}) \geq \limsup _{t \rightarrow \infty} \mathbb{P}\left(X_{t} \in[0, \delta]\right) \geq \mathbb{P}\left(\Omega_{0}\right)>0 .
$$

By the arbitrariness of $\delta$, we have $\pi^{\varphi}(\{0\} \times \mathcal{S})>\mathbb{P}\left(\Omega_{0}\right)>0$ which contradicts the fact that $\pi^{\varphi}$ is a probability measure on $(0, \infty) \times \mathcal{S}$ (see Lemma 2.3). We get the desired conclusion.

Proposition 2.7 If $\sum_{i \in \mathcal{S}} \mu_{i}\left(a_{1}(i)-\frac{1}{2} \alpha^{2}(i)\right)>0, \sum_{i \in \mathcal{S}} \mu_{i}\left(a_{2}(i)+\frac{1}{2} \beta^{2}(i)-\frac{c_{2}(i)}{m_{2}(i)}\right)<0$, and $\bar{\lambda}>0$, where $\bar{\lambda}$ is defined by (1.7), then $\lim \sup X_{t}>0$ a.s., $\lim \sup Y_{t}>0$ a.s. and $\left(X_{t}, Y_{t}, \Lambda_{t}\right)$ has a stationary distribution.

Proof. Since almost surely $X_{t} \leq \varphi_{t}$ and $Y_{t} \leq \psi_{t}$ for any $t>0$, the strong ergodicity theorem yields that almost surely

$$
\begin{gathered}
\limsup _{t \rightarrow \infty} \frac{1}{t} \ln X_{t} \leq \limsup _{t \rightarrow \infty} \frac{1}{t} \ln \varphi_{t} \leq \sum_{i \in \mathcal{S}} \mu_{i}\left(a_{1}(i)-\frac{1}{2} \alpha^{2}(i)\right)-\sum_{i \in \mathcal{S}} \int_{\mathbb{R}_{+}} b_{1}(i) x \pi^{\varphi}(\mathrm{d} x, i), \\
\limsup _{t \rightarrow \infty} \frac{1}{t} \ln Y_{t} \leq \limsup _{t \rightarrow \infty} \frac{1}{t} \ln \psi_{t} \leq-\sum_{i \in \mathcal{S}} \mu_{i}\left(a_{2}(i)+\frac{1}{2} \beta^{2}(i)-\frac{c_{2}(i)}{m_{2}(i)}\right) \\
-\sum_{i \in \mathcal{S}} \int_{\mathbb{R}_{+}} b_{2}(i) y \pi^{\psi}(\mathrm{d} y, i) .
\end{gathered}
$$

Next, we apply the trick used in [6, Theorem 2.2] to estimate the lower bounds.

$$
\begin{aligned}
\frac{1}{t} \ln X_{t}= & \frac{1}{t} \ln x_{0}+\frac{1}{t} \int_{0}^{t}\left(a_{1}\left(\Lambda_{s}\right)-\frac{1}{2} \alpha^{2}\left(\Lambda_{s}\right)-b_{1}\left(\Lambda_{s}\right) \varphi_{s}\right) \mathrm{d} s \\
& +\frac{1}{t} \int_{0}^{t}\left(b_{1}\left(\Lambda_{s}\right)\left(\varphi_{s}-X_{s}\right)-\frac{c_{1}\left(\Lambda_{s}\right) Y_{s}}{m_{1}\left(\Lambda_{s}\right)+m_{2}\left(\Lambda_{s}\right) X_{s}+m_{3}\left(\Lambda_{s}\right) Y_{s}}\right) \mathrm{d} s+\frac{1}{t} \int_{0}^{t} \alpha\left(\Lambda_{s}\right) \mathrm{d} B_{1}(s) \\
\geq & \frac{1}{t} \ln x_{0}+\frac{1}{t} \int_{0}^{t}\left(a_{1}\left(\Lambda_{s}\right)-\frac{1}{2} \alpha^{2}\left(\Lambda_{s}\right)-b_{1}\left(\Lambda_{s}\right) \varphi_{s}\right) \mathrm{d} s \\
& +\frac{1}{t} \int_{0}^{t}\left(b_{1}\left(\Lambda_{s}\right)\left(\varphi_{s}-X_{s}\right)-\frac{c_{1}\left(\Lambda_{s}\right) Y_{s}}{m_{1}\left(\Lambda_{s}\right)}\right) \mathrm{d} s+\frac{1}{t} \int_{0}^{t} \alpha\left(\Lambda_{s}\right) \mathrm{d} B_{1}(s), \quad \text { a.s. }
\end{aligned}
$$

Combining this with (2.16), we get

$$
\liminf _{t \rightarrow \infty} \frac{1}{t} \int_{0}^{t}\left(b_{1}\left(\Lambda_{s}\right)\left(X_{s}-\varphi_{s}\right)+\frac{c_{1}\left(\Lambda_{s}\right) Y_{s}}{m_{1}\left(\Lambda_{s}\right)}\right) \mathrm{d} s \geq 0, \quad \text { a.s. }
$$

and further

$$
\liminf _{t \rightarrow \infty} \frac{1}{t} \int_{0}^{t}\left(\hat{b}_{1}\left(X_{s}-\varphi_{s}\right)+\frac{\check{c}_{1}}{\hat{m}_{1}} Y_{s}\right) \mathrm{d} s \geq 0, \quad \text { a.s. }
$$


For the process $\left(Y_{t}\right)$, we have

$$
\begin{aligned}
\frac{1}{t} \ln Y_{t}= & \frac{1}{t} \ln y_{0}-\frac{1}{t} \int_{0}^{t} b_{2}\left(\Lambda_{s}\right) Y_{s} \mathrm{~d} s-\frac{1}{t} \int_{0}^{t}\left(a_{2}\left(\Lambda_{s}\right)+\frac{1}{2} \beta^{2}\left(\Lambda_{s}\right)-\frac{c_{2}\left(\Lambda_{s}\right) \varphi_{s}}{m_{1}\left(\Lambda_{s}\right)+m_{2}\left(\Lambda_{s}\right) \varphi_{s}}\right) \mathrm{d} s \\
& -\frac{1}{t} \int_{0}^{t}\left(\frac{c_{2}\left(\Lambda_{s}\right) \varphi_{s}}{m_{1}\left(\Lambda_{s}\right)+m_{2}\left(\Lambda_{s}\right) \varphi_{s}}-\frac{c_{2}\left(\Lambda_{s}\right) X_{s}}{m_{1}\left(\Lambda_{s}\right)+m_{2}\left(\Lambda_{s}\right) X_{s}}\right) \mathrm{d} s \\
& -\frac{1}{t} \int_{0}^{t}\left(\frac{c_{2}\left(\Lambda_{s}\right) X_{s}}{m_{1}\left(\Lambda_{s}\right)+m_{2}\left(\Lambda_{s}\right) X_{s}}-\frac{c_{2}\left(\Lambda_{s}\right) X_{s}}{m_{1}\left(\Lambda_{s}\right)+m_{2}\left(\Lambda_{s}\right) X_{s}+m_{3}\left(\Lambda_{s}\right) Y_{s}}\right) \mathrm{d} s \\
& +\frac{1}{t} \int_{0}^{t} \beta\left(\Lambda_{s}\right) \mathrm{d} B_{2}(s) \\
\geq & \frac{1}{t} \ln y_{0}+\frac{1}{t} \int_{0}^{t}\left(-a_{2}\left(\Lambda_{s}\right)-\frac{1}{2} \beta^{2}\left(\Lambda_{s}\right)+\frac{c_{2}\left(\Lambda_{s}\right) \varphi_{s}}{m_{1}\left(\Lambda_{s}\right)+m_{2}\left(\Lambda_{s}\right) \varphi_{s}}\right) \mathrm{d} s \\
& -\frac{1}{t} \int_{0}^{t}\left(\frac{c_{2}\left(\Lambda_{s}\right)\left(\varphi_{s}-X_{s}\right)}{m_{1}\left(\Lambda_{s}\right)+m_{2}\left(\Lambda_{s}\right) \varphi_{s}}+\left(\frac{c_{2}\left(\Lambda_{s}\right) m_{3}\left(\Lambda_{s}\right)}{m_{1}\left(\Lambda_{s}\right) m_{2}\left(\Lambda_{s}\right)}+b_{2}\left(\Lambda_{s}\right)\right) Y_{s}\right) \mathrm{d} s \\
& +\frac{1}{t} \int_{0}^{t} \beta\left(\Lambda_{s}\right) \mathrm{d} B_{2}(s), \quad \text { a.s. }
\end{aligned}
$$

It follows that

$$
\begin{aligned}
& \liminf _{t \rightarrow \infty} \frac{1}{t} \int_{0}^{t}\left(\frac{c_{2}\left(\Lambda_{s}\right)\left(\varphi_{s}-X_{s}\right)}{m_{1}\left(\Lambda_{s}\right)+m_{2}\left(\Lambda_{s}\right) \varphi_{s}}+\left(\frac{c_{2}\left(\Lambda_{s}\right) m_{3}\left(\Lambda_{s}\right)}{m_{1}\left(\Lambda_{s}\right) m_{2}\left(\Lambda_{s}\right)}+b_{2}\left(\Lambda_{s}\right)\right) Y_{s}\right) \mathrm{d} s \\
& \geq \liminf _{t \rightarrow \infty} \frac{1}{t} \int_{0}^{t}\left(-a_{2}\left(\Lambda_{s}\right)-\frac{1}{2} \beta^{2}\left(\Lambda_{s}\right)+\frac{c_{2}\left(\Lambda_{s}\right) \varphi_{s}}{m_{1}\left(\Lambda_{s}\right)+m_{2}\left(\Lambda_{s}\right) \varphi_{s}}\right) \mathrm{d} s-\limsup _{t \rightarrow \infty} \frac{1}{t} \ln Y_{t}, \quad \text { a.s. }
\end{aligned}
$$

Invoking (2.17) and taking Proposition 2.4 and Lemma 2.3 into consideration, we deduce that

$$
\begin{aligned}
& \liminf _{t \rightarrow \infty} \frac{1}{t} \int_{0}^{t}\left(\frac{c_{2}\left(\Lambda_{s}\right)\left(\varphi_{s}-X_{s}\right)}{m_{1}\left(\Lambda_{s}\right)+m_{2}\left(\Lambda_{s}\right) \varphi_{s}}+\left(\frac{c_{2}\left(\Lambda_{s}\right) m_{3}\left(\Lambda_{s}\right)}{m_{1}\left(\Lambda_{s}\right) m_{2}\left(\Lambda_{s}\right)}+b_{2}\left(\Lambda_{s}\right)\right) Y_{s}\right) \mathrm{d} s \\
& \geq \lambda+\sum_{i \in \mathcal{S}} \mu_{i}\left(a_{2}(i)+\frac{1}{2} \beta^{2}(i)-\frac{c_{2}(i)}{m_{2}(i)}\right)+\sum_{i \in \mathcal{S}} \int_{\mathbb{R}_{+}} b_{2}(i) y \pi^{\psi}(\mathrm{d} y, i)=\bar{\lambda}, \quad \text { a.s. }
\end{aligned}
$$

and further that

$$
\liminf _{t \rightarrow \infty} \frac{1}{t} \int_{0}^{t}\left(\frac{\check{c}_{2}}{\hat{m}_{1}}\left(\varphi_{s}-X_{s}\right)+\left(\frac{\check{c}_{2} \check{m}_{3}}{\hat{m}_{1} \hat{m}_{2}}+\check{b}_{2}\right) Y_{s}\right) \mathrm{d} s \geq \bar{\lambda}, \text { a.s. }
$$

Dividing both sides of (2.18) and (2.21) by $\hat{b}_{1}$ and $\check{c}_{2} / \hat{m}_{1}$ respectively, and adding them side by side, we obtain

$$
\liminf _{t \rightarrow \infty} \frac{1}{t} \int_{0}^{t} Y_{s} \mathrm{~d} s \geq \frac{\hat{m}_{1}}{\check{c}_{2}}\left(\frac{\check{m}_{3}}{\hat{m}_{2}}+\frac{\check{b}_{2} \hat{m}_{1}}{\check{c}_{2}}+\frac{\check{c}_{1}}{\hat{m}_{1} \hat{b}_{1}}\right)^{-1} \bar{\lambda}, \quad \text { a.s. }
$$

Hence, if $\bar{\lambda}>0$, then the inequality (2.22) implies that $\lim \sup _{t \rightarrow \infty} Y_{t}>0$ a.s. 
Note the following facts:

$$
\mathrm{d} \ln Y_{t} \leq\left(-a_{2}\left(\Lambda_{t}\right)-\frac{1}{2} \beta^{2}\left(\Lambda_{t}\right)+\frac{c_{2}\left(\Lambda_{t}\right) X_{t}}{m_{1}\left(\Lambda_{t}\right)+m_{2}\left(\Lambda_{t}\right) X_{t}+m_{3}\left(\Lambda_{t}\right) Y_{t}}\right) \mathrm{d} t+\beta\left(\Lambda_{t}\right) \mathrm{d} B_{2}(t),
$$

and $\lim _{t \rightarrow \infty} \frac{1}{t} \int_{0}^{t} \beta\left(\Lambda_{s}\right) \mathrm{d} B_{2}(s)=0$ a.s. If $\mathbb{P}\left(\left\{\omega: \lim _{t \rightarrow \infty} X_{t}(\omega)=0\right\}\right)>0$, then it must hold

$$
\mathbb{P}\left(\limsup _{t \rightarrow \infty} \frac{1}{t} \ln Y_{t}<0\right)>0 \text {. }
$$

Hence, the fact that $\lim \sup _{t \rightarrow \infty} Y_{t}>0$ a.s. implies that $\lim _{\sup _{t \rightarrow \infty}} X_{t}>0$ a.s.

At last, we show the existence of an invariant probability measure of the process $\left(X_{t}, Y_{t}, \Lambda_{t}\right)$. Note that almost surely $X_{t} \leq \varphi_{t}$ and $Y_{t} \leq \psi_{t}$ for any $t>0$. By (2.4) and (2.5) of Lemma 2.1, we deduce that for any $p>1$ satisfying $-\hat{a}_{2}+\frac{\check{c}_{2}}{\hat{m}_{2}}+\frac{p-1}{2} \check{\beta}^{2}>0$, there exists a constant $C>0$ such that

$$
\frac{1}{t} \int_{0}^{t} \mathbb{E}\left(X_{s}^{p}+Y_{s}^{p}+\Lambda_{s}^{p}\right) \mathrm{d} s \leq C .
$$

According to [3, Theorem 4.14], there exists a stationary distribution for $\left(X_{t}, Y_{t}, \Lambda_{t}\right)$. The proof is completed.

\section{Proof of Theorem 1.1}

The argument follows immediately from Propositions 2.3 , and 2.5, 2.7.

Remark 2.8 According to a suggestion of Hai Dang Nguyen, the condition (1.7) in Theorem 1.1 can be improved by noting that $\bar{\lambda}=\lambda$. We have showed that this equality holds when $N=1$. Actually it also holds for general $N>1$. By Itô's formula and the strong ergodic theorem,

$$
\lim _{t \rightarrow \infty} \frac{\ln \psi(t)}{t}=\lim _{t \rightarrow \infty} \frac{\ln \psi(0)}{t}+\bar{\lambda}-\lambda=\bar{\lambda}-\lambda .
$$

Since $(\psi(t))$ is ergodic in the current situation, then $\lim _{t \rightarrow \infty} \frac{\ln \psi(t)}{t}=0$, which yields $\bar{\lambda}=\lambda$.

Acknowledgment: The authors are very grateful to the referees for their valuable comments. The first author is supported by NSFC (No.11401592); The second author is supported by NSFC (No.11301030), NNSFC (No.11431014), 985-project.

\section{References}

[1] L. Arnold, W. Horsthemke, J.W. Stucki, The influence of external real and white noise on the Lotka-Volterra model, Biom. J., 21 (1979), 451-471. 
[2] G. K. Basak, A. Bisi, M. K. Ghosh, Stability of a random diffusion with linear drift, J. Math. Anal. Appl., 202 (1996), 604-622.

[3] M.-F. Chen, From Markov Chains to Non-equilibrium Particle Systems, World Scientific, 2nd edition, 2004.

[4] B. Cloez, M. Hairer, Exponential ergodicity for Markov processes with random switching, Bernoulli, 21 (2015), 505-536.

[5] N. H. Du, N. H. Dang, Dynamics of Kolmogorov systems of competitive type under the telegraph noise, J. Differential Equations, 250 (2011), 386-409.

[6] N. H. Du, N. H. Dang, G. Yin, Conditions for permanence and ergodicity of certain stochastic predator-prey models. to appear in J. Appl. Prob.

[7] R. Z. Khasminskii, Stochastic Stability of Differential Equations. Nauka, Moscow (1969). English transl.: Sijthoff and Noordhoff, Alphen, 1980

[8] J. Hofbauer, K. Sigmund, Evolutionary games and population dynamics, Cambridge University Press, 1998.

[9] N. Ikeda, S. Watanabe, Stochastic Differential Equations and Diffusion Processes, NorthHolland, New York, 1989.

[10] C. Ji, D. Jiang, Dynamics of a stochastic density dependent predator-prey system with Beddington-DeAngelis functional response, J. Math. Anal. Appl., 381 (2011), 441-453.

[11] R. Z. Khasminskii, F. C. Klebaner, Long term behavior of solutions of the Lotka-Volterra system under small random perturbations, Ann. Appl. Probab., 11 (2001), 952-963.

[12] M. Liu, K. Wang, Global stability of a nonlinear stochastic predator-prey system with Beddington-DeAngelis functional response, Commun. Nonlinear Sci. Numer. Simulat., 16 (2011), 1114-1121.

[13] M. Liu, K. Wang, The threshold between permanence and extinction for a stochastic Logistic model with regime switching, J. Appl. Math. Comput., 43 (2013), 329-349.

[14] S. Li, X. Zhang, Dynamics of a stochastic non-autonomous predator-prey system with Beddington-DeAngelis functional response, Adv. Difference Equ., 2013, 2013:19.

[15] X. Li, A. Gray, D. Jiang, X. Mao, Sufficient and necessary conditions of stochastic permanence and extinction for stochastic logistic populations under regime switching, J. Math. Anal. Appl., 376 (2011), 11-28. 
[16] X. Mao, Stochastic differential equations and applications, Horwood Publishing Limited, 1997.

[17] X. Mao, C. Yuan, Stochastic Differential Equations with Markovian Switching, Imperial College Press, London, 2006.

[18] M. Pinsky, R. Pinsky, Transience recurrence and central limit theorem behavior for diffusions in random temporal environments, Ann. Probab. 21 (1993), 433-452.

[19] R. Pinsky, M. Scheutzow, Some remarks and examples concerning the transience and recurrence of random diffusions. Ann. Inst. Henri. Poincaré , 28 (1992), 519-536.

[20] J. Shao, Ergodicity of regime-switching diffusions in Wasserstein distances, Stoch. Proc. Appl., 125 (2015), 739-758.

[21] J. Shao, Criteria for transience and recurrence of regime-switching diffusion processes, Electron. J. Probab., 20 (2015), 1-15.

[22] J. Shao, Ergodicity of one-dimensional regime-switching diffusion processes, Science China Math., 57 (2014), 2407-2414.

[23] J. Shao, F. B. Xi, Strong ergodicity of the regime-switching diffusion processes, Stoch. Proc. Appl., 123 (2013), 3903-3918.

[24] J. Shao, F. B. Xi, Stability and recurrence of regime-switching diffusion processes, SIAM J. Control Optim., 52 (2014), 3496-3516.

[25] A. V. Skorokhod, Asymptotic methods in the theory of stochastic differential equations, Vol. 78, American Mathematical Soc., 1989.

[26] G. G. Yin, C. Zhu, Hybrid switching diffusions: properties and applications, Vol. 63, Stochastic Modeling and Applied Probability, Springer, New York, 2010.

[27] C. Yuan, X. Mao, J. Lygeros, Stochastic hybrid delay population dynamics: well-posed models and extinction, J. Biol. Dyn., 3 (2009), 1-21.

[28] C. Zhu, G. Yin, On competitive Lotka-Volterra model in random environments, J. Math. Anal. Appl., 357 (2009), 154-170.

[29] C. Zhu, G. Yin, On hybrid competitive Lotka-Volterra ecosystems, Nonlinear Anal., 71 (2009), e1370-e1379. 